\title{
AI Based Predictive Model for Detection of Corona Patient
}

\author{
Dr. Santosh Kumar Sahoo \\ Assoc. Professor, CVR College of Engineering/ EIE Department, Hyderabad, India \\ Email: santosh.kr.sahoo@cvr.ac.in
}

\begin{abstract}
The proposed system can detect likelihood of the respiratory diseases, measure oxygen saturation and temperature of the patient. One of its goals is to test the patient for COVID-19 (i.e. pneumonia like symptoms). The system includes a testing stand which consists of respiratory sound recording unit, a pulse oximeter unit, an IR temperature check unit and a computer which consists of a software unit. The respiratory sound recording unit includes stethoscopes mounted inside a pillow on the testing stand which are connected to a microphone module which is further connected to the computer via an audio cable. The pulse oximeter unit includes a pulse oximeter sensor which is mounted on the right arm of the testing stand which is further connected to an Organic-LED (OLED) display, buzzer and the computer. The IR temperature check unit includes an IR temperature sensor which is mounted on the top arm of the testing stand which is further connected to an OLED display, buzzer and computer. It is to be noted that all the units can be adjusted according to the height of the patient. The software unit includes a machine learning program i.e. COVID-19 diagnostic management system, present in the computer which is connected with all the above-mentioned parameters.
\end{abstract}

Index Terms: Corona virus, Chest X-ray Radiographs, Artificial Intelligence, Deep Learning, CT Scan image

\section{INTRODUCTION}

The whole world is currently facing a health crisis in the form of Novel corona virus (2019- nCoV) outbreak and every country is facing challenges such as testing, quarantining and treating people affected by corona virus. 15 Chinese public health authorities on $31^{\text {st }}$ December 2019 reported Severe Acute Respiratory Syndrome (SARS) like pneumonia cases [1] of unidentified aetiology in the city of Wuhan, Hubei Province, China. It was later identified as a novel corona virus $(2019-\mathrm{nCoV})$ and it was the causative agent of this outbreak, now known as corona virus disease 2019 (COVID-19). The virus, 2019-nCoV is a 20-novel strain of corona virus, not identified previously in humans. The initial outburst of COVID-19 in Wuhan spread briskly and greatly affected other parts of China [1]. The spread was so rapid that it took the shape of an epidemic in no time, with coverage in several other countries. Unlike Severe Acute Respiratory Syndrome (SARS), the range of 2019$\mathrm{nCoV}$ epidemic is considerably wider. According to the European Centre for Disease Prevention and Control, more than 89,000 people globally were affected till March 2, 2020 and this number is increasing at an amazing rate, posing major hurdles for public health and the world economy.
World Health 30 Organization (WHO) has declared 2019$\mathrm{nCoV}$ outbreak as a public health emergency of international concern (PHEIC) on January 30, 2020 and a three pandemic on March 11, 2020. WHO, while declaring it as pandemic on March 11, 2020 also announced COVID-19 as name for the new corona virus disease [2],[3]. This is historic, as WHO had declared merely two pandemics in the past: in 1918 for influenza and in 2009 for influenza H1N1. According to WHO, the official 5 numbers count as of March 16, 2020 is more than 167,000 cases in at least 150 countries with more than 6606 deaths worldwide. WHO has also released the Corona virus disease (COVID-19) advice for the public and it can be accessed through WHO website. COVID-19 testing involves analyzing samples to assess the current or past presence of SARS-CoV-2. The two main branches detect either the presence of the virus or of antibodies produced in response to infection. Tests for viral presence are used to diagnose individual cases and to allow public health authorities to trace and contain outbreaks [4]. Antibody tests instead, show whether an individual was affected with the disease. They are less useful for diagnosing current infections because antibodies may not develop for weeks after infection. It is used to assess disease prevalence, which aids the estimation of the infection fatality rate [5]. Using a stethoscope, the doctor may hear normal breathing sounds, decreased or absent breath sounds, and abnormal breath sounds. If the doctor is unable to understand the problem clearly, they might suggest taking an X-ray or CT Scan. Both have quite long procedures, they are slow, costly, and takes time to get the results [6]. In the case of corona virus, for most patients, COVID-19 begins and ends in their lungs, because like the flu, corona virus are respiratory diseases. Currently, the types of tests for COVID-19 are Polymerase Chain Reaction (PCR) swab test, antibody blood test and in some cases, X-ray or CT Scan is recommended too. One researcher found COVID preventive automated entry point (COPAEP) is an integrated system which incorporates a definite number of subsystems each enabling checks and/or preventive actions against the most obvious symptoms of corona virus [7]. The research when deployed at the entry point of the place of human assembly restricts the virus-infected people from entering the premise; and hence, mixing of the Covid-infected population and the 
fit ones can be prevented to lower the further spread of corona virus. The system includes methods for measuring the body-temperature and coughing of the entrant. Second researcher found COVID-19 kit: intelligent thermal scanning kit for testing the COVID-19 positivity and displaying real time count of positive case using IoT Support structures for positioning sensors on a physiologic tunnel for measuring physical, chemical and biological parameters of the body and to produce an action according to the measured value of the parameters [8] [9]. Third researcher found corona virus (COVID-19) diagnostic reagents kits. This research relates to nucleic acids and proteins from the COVID-19 corona virus. These nucleic acids and proteins can be used in the preparation and manufacture of vaccine formulations, diagnostic reagents, kits, etc Fourth researcher studied development of a heartbeat and temperature measuring system for remote health nursing during pandemic (COVID-19). The device is implemented for monitoring of heartbeat and body temperature of patient based on microcontroller using the fingertip sensor device and temperature sensor. There are few drawbacks in the existing diagnostic system. Thus, to overcome the problems, there is need to develop an improved, effective and efficient system. Hence the present research provides a COVID-19 diagnostic system using artificial intelligence and machine learning [10].

\section{Model Description And Result}

The primary object of the present research is to provide Covid-19 diagnostic system using artificial intelligence and machine learning, which precisely detect likelihood of the Covid-19 [11]. Another object of the present research is to diagnose the patients for Covid-19 testing through respiratory sounds, oxygen saturation and temperature. Yet another object of the present research is to diagnose the patients with minimum contraction among healthcare providers and patients. Yet another object of the present research, is to quickly diagnose or test the patient which accelerates mass testing. Other objects, features and advantages will become apparent from detail description and appended claims to those skilled in art [12].

This work is described by way of example with reference to the following drawing where, Figure 1(A) shows covid19 diagnostic system with a patient, where 100 denotes respiratory sound recording unit, 101 denotes computer, 102 denotes pulse oximeter unit, 103 denotes IR temperature check unit, 104 denotes testing stand, 105 denotes pillow, 106 denotes software unit / covid-19 diagnostic management system, 107 denotes audio cable. Figure 1B shows expanded view of covid-19 diagnostic system, where 100 denotes respiratory sound recording unit, 101 denotes computer, 102 denotes pulse oximeter unit, 103 denotes IR temperature check unit, 104 denotes testing stand, 105 denotes pillow, 106 denotes software unit / COVID-19 diagnostic management system, 107 denotes audio cable, 108 denotes display unit [13]. Figure 2 demonstrates the block diagram of COVID-19 diagnostic system, where 200 denotes reset button, 201 denotes microcontroller, 202 denotes hardware code, 203 denotes stethoscope's microphone, 204 denotes pulse oximeter, 205 denotes IR temperature sensor, 206 denotes computer system, 207 denotes OLED display, 208 denotes buzzer, Figure 3 of represents the flow chart of procedure of COVID-19 diagnostic system. Figure 4 describes the final test reports (Figure 4(A), \& 4(B)) generated by the Covid COVID-19 diagnostic management system [14] [15]. The manner in which the above-cited and other advantages and objects of the research are obtained, a more detailed description of the research briefly described above, will be referred to, which is illustrated in the appended drawing. Understanding that these drawings depict only typical embodiment of the research and therefore not to be considered limiting in its scope, the research will be described with additional specificity and details through the use of the accompanying drawing 6 Detailed description of research: The present research relates to COVID-19 diagnostic system using artificial intelligence and machine learning which precisely detects likelihood of the COVID19. It comprises of a testing stand which consists of a respiratory sound recording unit, a pulse oximeter unit, an IR temperature check unit and a computer which consists of a software unit. Herein, the respiratory sound recording unit includes stethoscopes mounted inside a pillow which are connected to a microphone module which is further connected to the computer via an audio cable, the pulse oximeter unit includes a pulse oximeter sensor which is mounted on the right arm of the testing stand which is further connected to an OLED display, a buzzer and the computer and the IR temperature check unit includes an IR temperature sensor, which is mounted on the top arm of the testing stand which is further connected to an OLED display, a buzzer and the computer [16]. In this present task all the units can be adjusted according to the height of the patient. The software unit includes a machine learning program i.e. COVID-19 Diagnostic Management System, present in the computer which is connected to all the abovementioned parameters. Here, system can detect respiratory diseases, measure oxygen saturation and temperature of the patient [17]. For recording the respiratory sound of the patient, the patient needs to stand in front of the testing stand in such a way that his back is facing the testing stand. The patient should make sure that his back is in contact with the pillow present on the testing stand. The height of the pillow can be manually adjusted according to the height and comfort of the patient. Then, the patient should take deep breaths for 30 seconds and relax. Meanwhile, the respiratory recording unit records the respiratory sounds and transmits it to the software present in the computer. For the 
measurement of oxygen saturation level of the patient, the patient should make sure that his/her finger is gently placed on the red light (i.e. pulse oximeter sensor) present on the right arm of the testing stand [18]. The angle of the arm can be manually adjusted according to comfort of the patient. The patient must maintain his/her finger placement position (102) for at least 10 seconds. Meanwhile, the pulse oximeter unit measures the oxygen saturation and transmits it to the software present $\mathrm{n}$ the computer. For measurement of temperature of the patient, the patient should make sure that his head is next to the IR Temperature sensor which is present at the top arm of the testing stand. However, the position of the sensor can be manually adjusted according to the height and comfort of the patient. The IR temperature sensor measures the temperature within seconds.

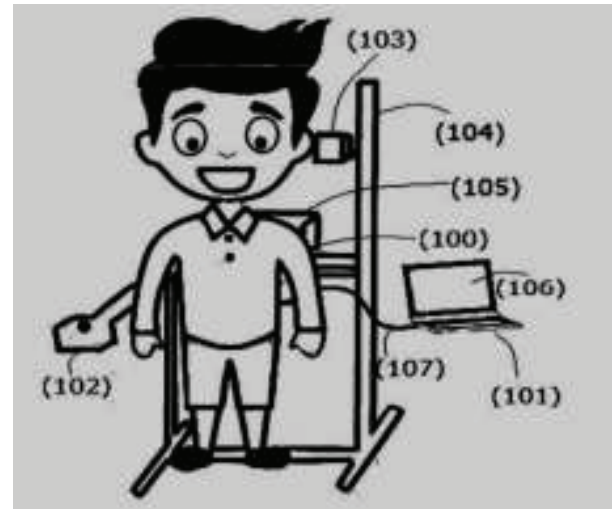

Figure 1(A). Covid-19 diagnostic system

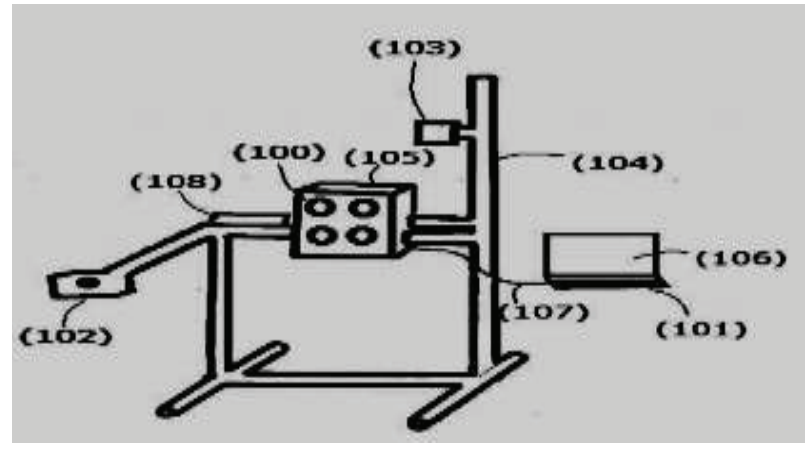

Figure 1(B). Expanded view of covid-19 diagnostic system

Meanwhile, the IR temperature sensor will transmit the data to the software present in the computer. In the present research as soon as the machine learning software i.e. COVID-19 Diagnostic Management System present in the computer receives the recordings and measurements, it will analyze them with its database through the AI based predictive model attached as per Figure 2 and Figure (2a).

This process will take a few seconds and will give out the final test result of the patient. Basically, the results will be displayed to the patient within a few minutes after their test. Therefore, the research in its broader aspect is not limited to specific details and representative embodiments shown and described herein. Accordingly, various modifications may be made without departing from the spirit or scope of the general work concept as defined by the appended claims and their equivalents.

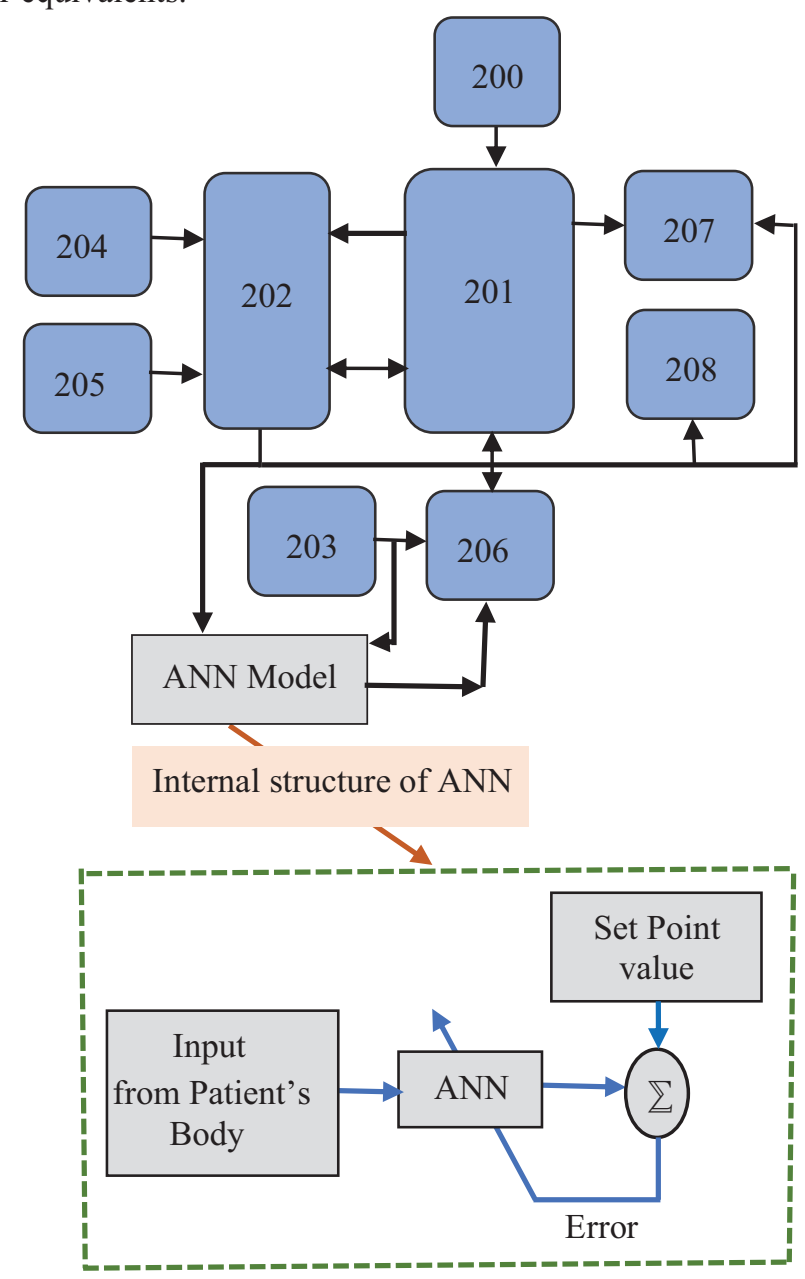

Figure 2. Block diagram of AI Based COVID-19 Predictive Model

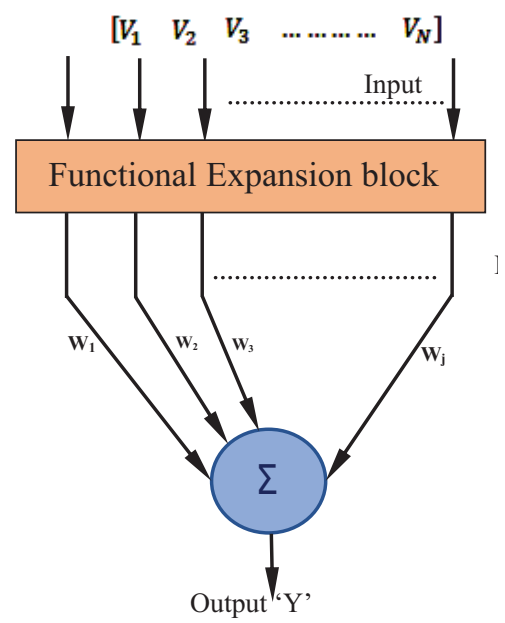

Figure 2(a): Simplified Functional ANN structure

In this article a trigonometric functional expansion is preferred for Fun-LANN. If all the polynomials of $x^{\text {th }}$ order with respect to an orthogonal system, then the best approximation in the metric space is presented by the partial summation of Fourier series of $\mathrm{x}^{\text {th }}$ polynomial with respect to system. 
Hence the trigonometric polynomial basis function is expressed as per equation (1).

$M=\{V, \operatorname{Sin}(\pi V), \operatorname{Cos}(\pi V), \operatorname{Sin}(2 \pi V), \operatorname{Cos}(2 \pi V) \ldots \ldots \operatorname{Sin}(x \pi V), \operatorname{Cos}(x \pi V)\}$

The above equation (1) signifies the compact representation of the function in mean square sense.

Let the Predictive model output is functionally expanded as ' $\mathrm{V}_{\text {Out }}$ '. The functionally expanded ' $\mathrm{M}$ 'vector is linearly combined with weight matrix by following means of equation (2).

$$
Y=\sum_{j=1}^{N} M_{j} w_{j}+\alpha
$$

Where $\alpha=$ bias term and $\boldsymbol{M}_{j}=\left\{\begin{array}{cr}V_{o} & j=1 \\ \sin \left(l \pi V_{o}\right), & j>1, j \text { even } \\ \cos \left(l \pi V_{o}\right), & j>1, j \text { odd }\end{array}\right.$

Where $1 \leq l \leq x, x$ is the order of expansion. Now the error signal can be defined as shown in equation (3).

$E(n)=D(n)-\sum_{i=1}^{N} M_{i} w_{i}$

The patient on Standing mode in front of predictive model

The patient's finger should be contact with pulse oximeter present on the right arm of testing stand

Patient is exposed to IR temperature sensor

Recording of parameter is started with proper instruction

The testing machine will record all the readings and analyze them using Covid-19 predictive model simulation platform

To get final test report click on "Get results"

Figure 3. Flow chart of Covid-19 diagnostic system

Where $\mathbf{D}(\mathrm{n})$ is set point value or desired signal or equivalent control signal applied to displacement actuator and ' $n$ ' is time index. Let the cost function ' $\zeta$ ' as residual noise power and it is presented as in equation (4).

$\zeta=\epsilon\left[E^{2}(n)\right]$

The weight vector $w_{j}$ may be adjusted as per following in order to optimization of mean square error (MSE) $\zeta$ in equation (5).

$w_{j+1}=w_{j}-\frac{\mu}{2} \nabla(\mathrm{n})$

Where $\nabla(\mathbf{n})$ is an instantaneous estimate of gradient of $\zeta$ with respect to weight vector $\boldsymbol{w}_{\boldsymbol{j}}$. Presented in equation (6).

Hence $w_{j+1}=w_{j}+\mu E(n) M(n)$

Where ' $\mu$ ' denotes the step size and it controls the convergence speed of the algorithm.
Date : 28-01-2021

Time : $21: 38: 3$

Patient' Name : ABCX

Age : 25

Gender : Male

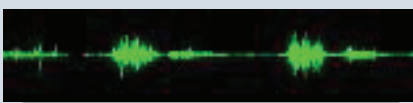

\begin{tabular}{|c|c|}
\hline Oxygen $\%$ & Temperature \\
\hline $90 \%$ & $98.6^{0} \mathrm{~F}$ \\
\hline
\end{tabular}

Suspected Disease/ Condition

Normal

Figure 4(A). Final test reports

Date : $28-01-2021$

Time : $21: 38: 3$

Patient' Name : ABCX

Age : 25

Gender

: Male

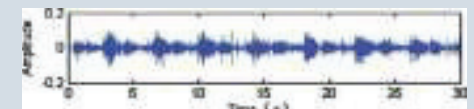

\begin{tabular}{|c|c|}
\hline Oxygen \% & Temperature \\
\hline $97 \%$ & $100.6^{0} \mathrm{~F}$ \\
\hline
\end{tabular}

Suspected Disease/ Condition

Wheezes

Figure 4 (B). Final test reports generated by the Covid-19 diagnostic management system

\section{CONCLUSIONS}

In the present work the machine learning software based COVID-19 Diagnostic Management System receives the information from the corresponding patients, and it will record as well as analyze them. This process will take a few seconds and will provide the final test results of the patient. The results will be displayed to the patient within a few minutes after their test. Additional advantages and modification will readily occur to those skilled in the art.

Therefore, the work in its broader aspect is not limited to specific details and representative embodiments described herein. Accordingly, various modifications may be made 
without departing from the spirit or scope of the general research concept as defined by the appended objective and their equivalents.

\section{REFERENCES}

[1] Ruiz Estrada MA. The uses of drones in case of massive Epidemics contagious diseases relief humanitarian aid: Wuhan-COVID-19 crisis. SSRN Electron J 2020.

[2] Nguyen TT, Waurn G, Campus P. Artificial intelligence in the battle against corona virus (COVID-19): A survey and future research directions. 2020

[3] Maghdid HS, Ghafoor KZ, Sadiq AS, Curran K, Rabie K. A novel AI-enabled framework to diagnose coronavirus COVID 19 using smartphone embedded sensors: design study. 2020. page. 1-5.

[4] Wang CJ, New T, Sun F, et al. Response to COVID-19 in Taiwan big data analytics, new technology, and proactive testing. 2020. p. 1-2.

[5] Aishwarya Kumar, Puneet Kumar Gupta*, Ankita Srivastava, A review of modern technologies for tackling COVID-19 pandemic, Elsevier, Diabetes \& Metabolic Syndrome: Clinical Research \& Reviews 14 (2020) pg -569573

[6] Ai T, Yang Z, Hou H, Zhan C, Chen C, Lv W, Tao Q, Sun Z, Xia L. Correlation of chest CT and RT-PCR testing in corona virus disease 2019 (COVID-19) in China: a report of 1014 cases. Radiology 2020.

[7] Luo H, Tang QL, Shang YX, Liang SB, Yang M, Robinson N, Liu JP. Can Chinese medicine be used for prevention of coronavirus disease 2019 (COVID-19)? A review of historical classics, research evidence and current prevention programs. Chin J Integr Med 2020.

[8] Haleem A, Vaishya R, Javaid M, Khan IH. Artificial Intelligence (AI) applications in orthopaedics: an innovative technology to embrace. J Clin Orthop Trauma 2019.

[9] Stebbing J, Phelan A, Griffin I, Tucker C, Oechsle O, Smith D, Richardson P. COVID-19: combining antiviral and antiinflammatory treatments. Lancet Infect Dis 2020 Feb 27.

[10] Raju Vaishya, Mohd Javaid, Ibrahim Haleem Khan, Abid Haleem, Artificial Intelligence (AI) applications for COVID-19 pandemic, Elsevier, Diabetes \& Metabolic Syndrome: Clinical Research \& Reviews 14 (2020) Page 337-339

[11] Pedro R. A. S. Bassi, Romis Attux, A Deep Convolutional Neural Network for COVID-19 Detection Using Chest XRays, arXiv:2005.01578v2[eess.2], 18 june 2020, page - 119

[12] Asif Iqbal Khan, Junaid Latief Shah, Mohammad Mudasir Bhat, CoroNet: A deep neural network for detection and diagnosis of COVID-19 from chest x-ray images, Elsevier, Computer Methods and Programs in Biomedicine 196 (2020) 105581, Page 1-9

[13] Lin Li, BS Lixin Qin, PhD • Zeguo Xu, BS • Youbing Yin, $\mathrm{PhD} \cdot \mathrm{X}$ in Wang, $\mathrm{PhD} \bullet$ Bin Kong, $\mathrm{PhD} \cdot$ Junjie Bai, $\mathrm{PhD} \bullet$ Yi Lu, MS • Zhenghan Fang, MS • Qi Song, PhD • Kunlin Cao, $\mathrm{PhD} \cdot$ Daliang Liu, PhD • Guisheng Wang, PhD • Qizhong Xu, MS • Xisheng Fang, BS • Shiqin Zhang, BS •
Juan Xia, BS • Jun Xia, PhD, Using Artificial Intelligence to Detect COVID-19 and Community-acquired Pneumonia Based on Pulmonary CT, Radiology 2020.

[14] Clinical Trials.gov. Bethesda (MD): National Library of Medicine (US). Identifier NCT03945825, Flublok or Fluzone With Advax-CpG55.2 or AF03; 2020 March 16 [cited 2020 March 23];

[15] Park A. AI develops' turbocharged' flu vaccine: A clinical trial will soon be underway in the U.S. to test the efficacy of an enhanced flu vaccine developed by an artificial intelligence system at Flinders University in South Australia. Becker's Hospital Review. Accessed March 24, 2020.

[16] Smith M, Smith JC. Repurposing therapeutics for COVID19: Supercomputer-based docking to the SARS-CoV-2 viral Spike Protein and viral spike protein-human ACE2 interface. Chem Rxiv 2020,

[17] Artificial intelligence and COVID-19: A multidisciplinary approach, Integrative Medicine Research 9 (2020) 100434

[18] Pravin RK, Akojwar SG, Bajaj ND. 2018. A hybridized neural network and optimization algorithms for prediction and classification of neurological disorders. Int $\mathrm{J}$ Biomed Eng Technol. 28(4):307-321. 